\title{
Nanometer Scale Atomic Order in a Bulk Metallic Glass from Fluctuation Microscopy
}

\author{
Jinwoo Hwang, and P. M. Voyles \\ Department of Materials Science and Engineering, University of Wisconsin, Madison, \\ Madison, WI 53706
}

In bulk metallic glasses (BMGs), nanometer-scale atomic structure has been proposed to influence the geometric frustration [1], and to play a central role in the creation and activation of the fundamental flow defect, shear transformation zones [2]. Recent structural studies $[3,4]$ have suggested various forms of medium-range order (MRO) that extend in length scale up to a few nanometers, in addition to the known short-range order (SRO) in BMGs, but a general, experimentally-tested structural model has not yet been found.

We have studied the structure of ternary $\mathrm{Zr}-\mathrm{Cu}-\mathrm{Al}$ BMGs using variable coherence fluctuation electron microscopy (FEM) [5] and electron diffraction [6]. Electron diffraction yields the total electron reduced density function $G(r)$, shown in Figure 1(a), which primarily carries information about SRO. FEM measures the variance, $V$, shown in Figure 1(b), of dark field images acquired at nanometer spatial resolution as a function of the scattering vector magnitude, $k . V(k)$ carries information about MRO.

Finding the atomic configurations that give rise to the $V(k)$ in Figure 1(b) is challenging. Unlike most previous materials studied by FEM, BMGs have nearly equal concentrations of several elements, and a large spectrum of possible structural motifs, including several related crystal phases and various non-crystalline polyhedra [3]. We have turned to reverse Monte Carlo (RMC) structural modeling [7], which uses random atomic moves to minimize the $\chi^{2}$ between simulated experiments and measured data. We generated $\sim 50,000$ atom RMC models refined against $G(r)$ alone and against both $G(r)$ and $V(k)$ [8]. In Figure 1(a), the $V(k)+G(r)$ and the only-G(r) models both show very similar agreement with the experimental $G(r)$. The disagreement around the first peak is due to an experimental artifact, which we believe is caused by an insufficient $k$-range in the diffraction experiment. In Figure 1(b), the $V(k)+G(r)$ model shows an excellent match with the experimental $V(k)$, but the only-G(r) model does not show any peaks, showing that including FEM data in RMC further constrained the model, increasing its uniqueness.

Starting from essentially zero initial $V$, RMC refinement increased the simulated $V(k)$ to match the experiment by creating loosely ordered nanoscale planar structures in the model. The ordered pseudo-planes diffract more strongly, increasing the intensity of some image pixels and increasing $V$, as shown in Figure 2(a). Figure 2(b) shows the projected pair distribution function and drawings of the atoms in a bright pixel and a dark pixel. The atoms in the bright pixel are ordered, and the atoms in dark pixel are not. Also in Figure 2(a), many pixels have lower intensity after RMC refinement, which is because RMC tends to produce the most disordered structure consistent with the data. 
These results suggest that the FEM $V(k)$ arises from nanometer scale plane-like order. The planar order regions in our models so far are aligned in only one direction, which is unreasonably anisotropic. There are a few regions with planes in more than one direction, suggesting that improved simulations will generate more realistic structures [9].

\section{References}

[1] D. Miracle, Nature Materials 3, 701 (2004).

[2] C. A. Schuh, T. C. Hufnagel, and U. Ramamurty, Acta Mater. 55, 4067 (2007).

[3] H. W. Sheng, W. K. Lou, F. M. Alamgir, J. M. Bai, and E. Ma, Nature 439, 419 (2006).

[4] D. Ma, A. D. Stoica, and X. -L. Wang, Nature Materials 8, 30 (2009).

[5] M. M. J. Treacy et al., Rep. Prog. Phys. 68, 2899-2944 (2005).

[6] D. J. H. Cockayne and D. R. McKenzie, Acta Cryst. A44, 870 (1988).

[7] D. A. Keen and R. L. Mcgreevy, Nature 344, 423-425 (1990); R. L. McGreevy, J. Phys.: Condens. Matter 13, R877 (2001).

[8] Jinwoo Hwang et al., Physical Review B (submitted).

[9] This work is supported by the National Science Foundation (CMMI- 0824719).
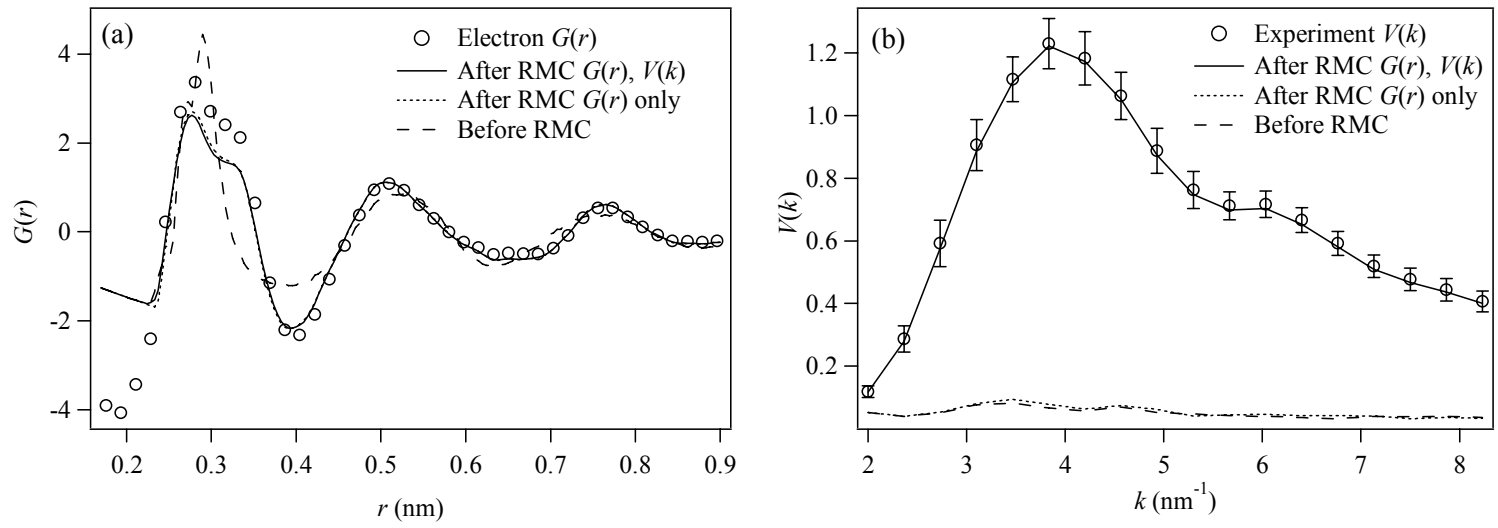

Figure 1. (a) Simulated $G(r)$ before and after RMC compared to the experimental $G(r)$. (b) Simulated $V(k)$ before and after RMC compared to the experimental $V(k)$.
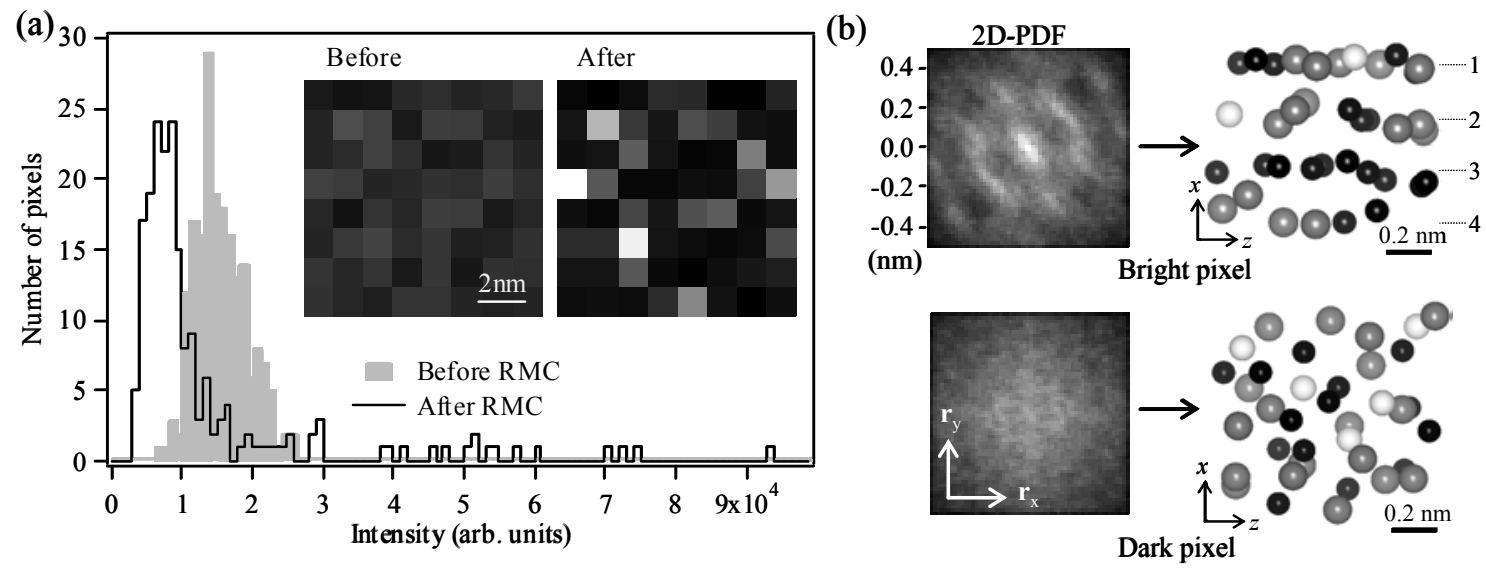

Figure 2. (a) Simulated images and their histograms before and after RMC. (b) 2D-PDFs and atom arrangements in the bright pixel and the dark pixel perpendicular to the beam direction. The gray, black, white atoms are $\mathrm{Zr}, \mathrm{Cu}$, and $\mathrm{Al}$, respectively. 